\title{
Genetic and Environmental Basis in Phenotype Correlation Between Physical Function and Cognition in Aging Chinese Twins
}

\author{
Chunsheng Xu, ${ }^{1,2}$ Dongfeng Zhang, ${ }^{1}$ Xiaocao Tian, ${ }^{2}$ Yili Wu, ${ }^{1}$ Zengchang Pang, ${ }^{1,2}$ Shuxia Li, ${ }^{3}$ \\ and Qihua $\operatorname{Tan}^{3,4}$ \\ ${ }^{1}$ Department of Epidemiology and Health Statistics, Qingdao University Medical College, Qingdao, Shandong, China \\ ${ }^{2}$ Qingdao Municipal Center for Disease Control and Prevention, Qingdao, Shandong, China \\ ${ }^{3}$ Unit of Human Genetics, Department of Clinical Research, University of Southern Denmark, Odense, Denmark \\ ${ }^{4}$ Epidemiology, Biostatistics and Biodemography, Department of Public Health, University of Southern Denmark, Odense, \\ Denmark
}

\begin{abstract}
Although the correlation between cognition and physical function has been well studied in the general population, the genetic and environmental nature of the correlation has been rarely investigated. We conducted a classical twin analysis on cognitive and physical function, including forced expiratory volume in one second $\left(\mathrm{FEV}_{1}\right)$, forced vital capacity $(\mathrm{FVC})$, handgrip strength, five-times-sit-to-stand test (FTSST), near visual acuity, and number of teeth lost in 379 complete twin pairs. Bivariate twin models were fitted to estimate the genetic and environmental correlation between physical and cognitive function. Bivariate analysis showed mildly positively genetic correlations between cognition and $\mathrm{FEV}_{1}, \mathrm{r}_{\mathrm{G}}=0.23$ [95\% $\left.\mathrm{Cl}: 0.03,0.62\right]$, as well as FVC, $r_{G}=0.35$ [95\% Cl: 0.06, 1.00]. We found that FTSST and cognition presented very high common environmental correlation, $r_{\mathrm{C}}=-1.00$ [95\% Cl: -1.00, -0.57], and low but significant unique environmental correlation, $r_{\mathrm{E}}=-0.11[95 \% \mathrm{Cl}:-0.22,-0.01]$, all in the negative direction. Meanwhile, near visual acuity and cognition also showed unique environmental correlation, $r_{\mathrm{E}}=0.16$ [95\% $\left.\mathrm{Cl}: 0.03,0.27\right]$. We found no significantly genetic correlation for cognition with handgrip strength, FTSST, near visual acuity, and number of teeth lost. Cognitive function was genetically related to pulmonary function. The FTSST and cognition shared almost the same common environmental factors but only part of the unique environmental factors, both with negative correlation. In contrast, near visual acuity and cognition may positively share part of the unique environmental factors.
\end{abstract}

Keywords: physical and cognitive function, genetic correlation, aging Chinese twins

Cognitive function and physical function, such as pulmonary, musculoskeletal, visual, and oral functions, are essential to guarantee the well-being, independent living, and quality of life in the elderly population. Pulmonary function is associated with mortality and aging-related diseases. Forced expiratory volume in one second $\left(\mathrm{FEV}_{1}\right)$, an indicator that tests airflow, and forced vital capacity (FVC), a parameter that measures lung volume, are the two most important and standard indicators of pulmonary function. Skeletal muscle strength is often considered as an important indicator of general vitality, which includes upper and lower limb strength. The upper limb strength can be assessed by handgrip strength and the lower limb may be represented by the five-times-sit-to-stand test (FTSST). Handgrip strength and the FTSST are the representatives of skeletal muscle strength, which is known to be associated with muscular functioning and activities of daily living (ADL) functioning (Rantanen et al., 2002). Besides, aging may lead to functional presbyopia for which near visual acuity is a crucial trait to assess the visual function. Oral function is necessary to maintain the chewing process, which may be assessed by dental health. The number of remaining teeth is essential to maintain basic nutritional supplying in the elderly. The self-reported number of teeth loss caused by natural aging is an easy way to assess the dental or oral

RECEIVED 10 October 2016; ACCEPTED 17 November 2016 ADDRESS FOR CORRESPONDENCE: Professor Dongfeng Zhang, No. 38 Dengzhou Road, Shibei District, Qingdao University Medical College, Qingdao, Shandong, 266021, China. E-mail: zhangdf1961@126.com 
function. $\mathrm{FEV}_{1}$, FVC, handgrip strength, FTSST, near visual acuity, and number of teeth loss are inexpensive, easily measurable, and preferred phenotypes and have been regarded as the important indicators of physical function for extending healthy life expectancy.

Human aging is a gradual degradation of physiological or biological process, which is accompanied by both physical and cognitive changes. The progressive decline in $\mathrm{FEV}_{1}$, FVC, handgrip strength, near visual acuity, and cognitive ability and increase in FTSST and number of teeth lost late in life are crucial issues in aspects of prevalence, morbidity, and functional limitations that severely affect the independent living arrangements of the elderly and therefore their quality of life.

Physical and cognitive functions are both important determinants in living independently in late life. Good physical fitness can help to reduce the risk of cognitive impairment and maintain cognitive function. The correlation between physical and cognitive function has been well studied in general populations. Cognitive function was associated with pulmonary function (Anstey et al., 2004; Emery et al., 2012; Feng et al., 2012), handgrip strength (Sternang et al., 2016), lower limb strength (Chen et al., 2015; Ohsugi et al., 2014), and number of teeth lost (Saito et al., 2013). However, the genetic and environmental bases for the correlation between cognitive function and near visual acuity have not been well studied (Mueller-Schotte et al., 2016).

Hagenaars et al. (2016) reported the shared genetic etiology between cognitive function and $\mathrm{FEV}_{1}$, one of the indicators of physical function, by linkage disequilibrium regression and polygenic profile scoring using the Biobank and GWAS consortia but drew no conclusion about the genetic correlation between them, possibly due to the method and population sample employed. Others (Johnson et al., 2009a, 2009b) reported the genetic and environmental influences on correlations between cognitive ability and physical fitness using self-reported capability in daily life-for example, walking, running, and climbing stairs. Emery et al. (1998) used the twin method to report that genetic factors accounted for a greater part in the correlation of $\mathrm{FEV}_{1}$ and cognitive function than environmental factors. We report here the first Chinese twin study aimed at assessing the genetic and environmental background in the correlation between indicators of physical and cognitive function using the classical twin design.

\section{Materials and Methods}

\section{Sample Collection}

The sample collection was from the Qingdao Twin Registry (QTR), the first twin registry in China (Li et al., 2006, 2013), established at the Qingdao Center for Disease Control and Prevention (Qingdao CDC; Duan et al., 2013; Pang et al., 2006). We recruited the twin subjects through the QTR and the local CDC, and via community and media publicity from 2012 to 2013 . Twins who suffered from pulmonary, skeletal muscle, visual, and dental diseases, as well as incomplete twin pairs, were excluded from sampling. We sampled a total of 379 complete adult twin pairs, including 240 monozygotic (MZ) twins (114 male [MZM] and 126 female $[\mathrm{MZF}]$ pairs) and 139 dizygotic (DZ) twins (41 male [DZM], 39 female [DZF], and 59 opposite sex [OSDZ] pairs) aged $51.8 \pm 7.6$ (40-80 years old). The collected twin samples did not represent a specific social class or education status. Zygosity was identified by DNA markers with $99.9 \%$ correct assignment (Becker et al., 1997; Tomsey et al., 2001) at the laboratory of Qingdao Blood Center.

Institutional Review Boards of the Qingdao CDC approved the study. We conducted the project in accordance with the ethical principles of the Helsinki Declaration. All voluntary twin subjects gave their informed consent and then completed the Montreal Cognitive Assessment (MoCA) questionnaire and physical examination, under the supervision of physicians at the Qingdao CDC.

\section{Measurements of Physical Function}

Pulmonary function was assessed by $\mathrm{FEV}_{1}$ and $\mathrm{FVC}$ (liter), measured using the electronic hand-held spirometer (Micro 0102). Every subject was tested two times following the standard procedure, and the best trial data were used for analysis. Handgrip strength (kilograms) and strength of upper limb were tested by the electronic dynamometer (WCS100, Nantong, China). Participants squeezed the handle as hard as possible in a standing position with their arms straight down. The maximum of three tests on each hand were adopted in the study. The FTSST was used to evaluate the strength of lower limb. The subjects were asked to fold their arms across their chests and rise from the chair and then sit down fully as fast as they could for five times without pause. The time spent to complete the test was recorded using a stop watch. Near visual acuity was measured with the standard near vision E chart (Beijing, China). The participants were asked to read the chart, held up at eye level and $30 \mathrm{~cm}$ away in natural outdoor light, without artificial visual assistance. The lower line of the chart that the subject could discern as much as possible by each eye was recorded to represent the higher near visual acuity. Dental function was measured by the number of teeth lost reported by the subjects themselves, not including those caused by trauma, dental treatment, and other situations unrelated to aging.

The measuring instruments, including spirometer, dynamometer, and stop watch, were calibrated every morning before use.

\section{Measurements of Cognitive Function}

The MoCA (www.mocatest.org) was adopted to evaluate the cognitive function of the participants. The MoCA includes several domains of cognition and is a good agesensitive and effective tool for cognitive screening (Gluhm et al., 2013; Luis et al., 2009; Nasreddine et al., 2005). 
Each twin pair was interviewed face to face by the same well-trained and experienced investigator. The score of the MoCA was adjusted to add 1 if the participant had no more than 12 years of schooling.

\section{Data Analysis}

Epidata3.1 (www.epidata.dk) was adopted to conduct the parallel input and correction of the data. The Box-Cox transformation was applied to normalize all the indicators before twin modeling. The Box-Cox transformation was done using the free $\mathrm{R}$ package car (http://cran. r-project.org/web/packages/car/index.html).

\section{Twin Modeling}

We performed bivariate twin modeling to dissect the correlation between cognitive and physical function to genetic correlation $\left(r_{\mathrm{G}}\right)$, common environmental correlation $\left(r_{\mathrm{C}}\right)$, and unique environmental correlation $\left(\mathrm{rr}_{\mathrm{E}}\right)$, to be estimated by the full ACE model. The nested models, such as $\mathrm{AE}, \mathrm{CE}$ and $\mathrm{AC}$, were fitted by dropping every component whose statistical significance was tested by the likelihood ratio test. The full and nested models were compared so that we could choose the best-fitting model on the basis of the parsimonious principle and Akaike's information criterion (AIC; Akaike, 1987), with the lowest value for the best-fitting model. The cross-twin, cross-trait correlation (CTCTC) was estimated before fitting the bivariate model and compared across zygosities to provide indication of genetic correlation between the two traits of interest. Age and sex were adjusted in all twin analyses. Twin models were fitted by the free Mx (http://www.vcu.edu/mx).

\section{Results}

The descriptive statistics of $\mathrm{FEV}_{1}$, FVC, handgrip, FTSST, and cognitive function have been described in detail elsewhere (Tian et al., 2017; Xu et al., 2015). There was a strong pattern of decline in $\mathrm{FEV}_{1}, \mathrm{FVC}$, handgrip strength, near visual acuity, and cognitive function, but gains in FTSST and number of teeth lost with increasing age, as indicated by the significantly negative and positive regression coefficients $(p<.05)$, respectively. Sex differences were observed for the indicators except for the FTSST, near visual acuity, and cognition, with females having significantly lower levels of $\mathrm{FEV}_{1}, \mathrm{FVC}$, handgrip strength and higher level of teeth lost than those of males. There were significant agegender interactions in $\mathrm{FEV}_{1}$, handgrip strength, and number of teeth lost, with positive or negative regression coefficients meaning that females outperformed males in $\mathrm{FEV}_{1}$ and handgrip strength, and underperformed in number of teeth lost with increasing age.

We found significant differences in CTCTC for cognitive function with $\mathrm{FEV}_{1}$, as well as with near visual acuity between MZ and DZ twins (Table 1). CTCTCs for six combinations were higher in MZ than those in DZ twins ex-

\section{TABLE 1}

Phenotypic Correlations (Cross-Twin Cross-Trait Correlation)

Between Physical and Cognitive Function Pairs

\begin{tabular}{lccccc}
\hline Indicators pair & $\mathrm{MZ}$ & $\mathrm{n}_{\mathrm{MZ}}$ & \multicolumn{1}{c}{$\mathrm{DZ}$} & $\mathrm{n}_{\mathrm{DZ}}$ & $p$ \\
\hline FEV $_{1}$ - cognition & 0.17 & 238 & -0.06 & 139 & .03 \\
FVC-cognition & 0.15 & 238 & 0.03 & 139 & .26 \\
Handgrip-cognition & 0.07 & 232 & -0.08 & 137 & .17 \\
FTSST-cognition & -0.29 & 235 & -0.24 & 136 & .62 \\
Near vision-cognition & 0.29 & 237 & 0.07 & 139 & .03 \\
Teeth lost-cognition & -0.15 & 238 & -0.10 & 139 & .64 \\
\hline
\end{tabular}

Note: MZ: monozygotic. DZ: dizygotic.

cept for handgrip strength, implying the potential genetic involvement in the correlations of cognition with $\mathrm{FEV}_{1}$, FVC, FTSST, near visual acuity, and the number of teeth lost. Bivariate twin models were then fitted to estimate the genetic and environmental correlations for cognition with the six indicators of physical function, followed by the procedure for best-fitting model selection. In Table 2, we show the estimated genetic and environmental variances, covariances and correlations in the full models, and the bestfitting models for the combination of cognitive function with every indicator of physical function.

The correlations of cognition with handgrip strength, as well as with FTSST, were best fitted by the CE model, whereas the correlations of cognition with the other four indicators of physical function were all best fitted by the $\mathrm{AE}$ model. The two indicators of pulmonary function, $\mathrm{FEV}_{1}$ and FVC, which had higher CTCTCs in MZ than those in DZ twins, also had significant genetic correlations $\left(r_{\mathrm{G}}\right)$ with cognition, all with 95\% CIs above zero. The mildly positively genetic correlation between $\mathrm{FEV}_{1}$ and cognition was $r_{\mathrm{G}}=0.23$ [95\% CI: 0.03, 0.62], and between FVC and cognition it was $r_{\mathrm{G}}=0.35$ [95\% CI: 0.06, 1.00]. Cognition had no significant genetic correlation with the other four indicators of physical function. We also found significant unique environmental correlation between cognition and FTSST, $r_{\mathrm{E}}=-0.11$ [95\% CI: $-0.22,-0.01$ ], and between cognition and near visual acuity, $r_{\mathrm{E}}=0.16$ [95\% CI: 0.03, 0.27], with opposite or same directions, respectively. Furthermore, we also observed negatively significant common environmental correlation between FTSST and cognition, $r_{\mathrm{C}}=-1.00$ [95\% CI: -1.00, -0.57]. The genetic, common, and unique environmental correlations of the other combinations were all zero.

\section{Discussion}

The genetic correlation expresses the extent to which two measurements reflect the same set of genes. The study of genetic correlation can facilitate exploration of the pleiotropic genetic variant. This study found that the relatedness between cognitive and pulmonary function stemmed predominantly from genetic rather than the environmental causes, implying that the underlying genes may have pleiotropic effects on $\mathrm{FEV}_{1}, \mathrm{FVC}$, and cognition. The 


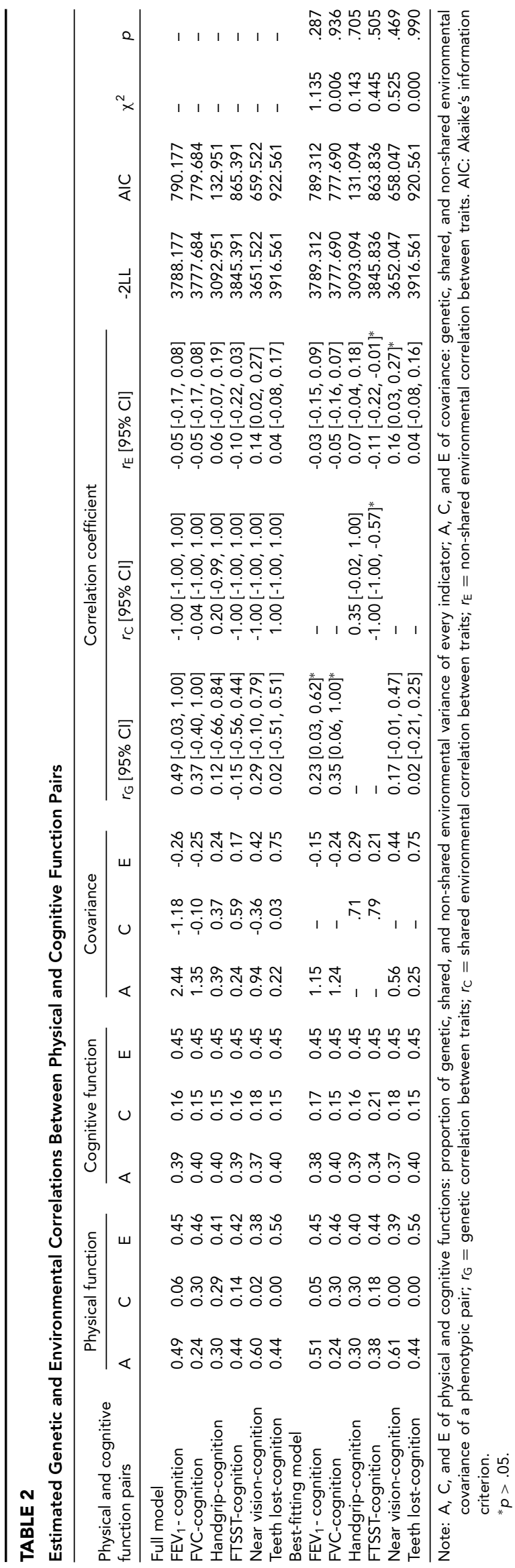

bivariate genetic analysis showed that the genetic correlation between $\mathrm{FEV}_{1}, \mathrm{FVC}$, and cognition was mild and in a positive direction, which was in line with the corresponding CTCTC estimate, suggesting that pulmonary and cognitive function may share a similar genetic basis that positively regulates the correlation between the two anthropometric features, which is consistent with the results of Emery et al. (1998). Molecular genetic studies should help to identify specific genes or genetic variants regulating both pulmonary and cognitive functions.

We did not find significant genetic correlation between cognition and the other four indicators of physical function. In our estimates, the correlation between handgrip strength and cognition was very low in both MZ (0.07) and DZ $(-0.08)$ twins. The weak correlation and the reversed pattern in $\mathrm{MZ}$ and $\mathrm{DZ}$ twins (DZ > MZ) did not support or suggest the existence of genetic involvement between them, which complied with the subsequent bivariate analysis that showed no genetic correlations between the two indicators. Although the CTCTCs for cognition with FTSST, near visual acuity, and number of teeth lost were higher in MZ than DZ twins, our twin modeling still failed to reveal the shared genetic basis between them. We concluded that although handgrip strength, FTSST, near visual acuity, number of teeth lost, and cognitive function presented moderate heritability, they may be modulated by their own genes and they were less likely to share similar genetic basis.

We found that the FTSST and cognition presented not only the perfect common environmental correlation, but also mild unique environmental correlations, both in the negative direction. The results could mean that common or unique environmental factors tending to extend the FTSST may reduce the cognitive function simultaneously and vice versa. Additionally, we found that near visual acuity and cognition mildly and positively shared the unique environmental factors, which could raise the level of near visual acuity and improve the cognitive function at the same time.

We would like to emphasize the importance of common environmental factors, such as socio-economic status, medical or healthcare availability, socio-cultural traditions, and so on, and the unique environmental factors-for example, individual habits, lifestyle choices, occupation, educational level, and income-in their effect on cognitive function (Cassarino \& Setti, 2015). The significant common and unique environmental correlations between the two forms of body function implied that good health and lifestyle habits could be beneficial to maintain both physical and cognitive functions with aging. Health education in the aging population is needed to cultivate good habits, regular physical exercise, and so on to facilitate normal functioning during aging.

There were several limitations of the study. First, the age of the present sample was relatively young compared to the Western studies and covered a large age span ( $40-80$ years). The non-linear effect of age-dependent change in physical 
and cognitive function, if any, could have been averaged out, which could possibly contribute to reducing the power of the study, although we adjusted the effect of age in twin modeling. Second, the sample size was relatively small compared with Western studies, which could lead to the wide 95\% confidence interval, as shown in Table 2. Third, the MoCA was just a screening instrument. It may be suboptimal to assess the participants' cognitive function, particularly for the younger half of the samples. We hoped that future studies with sizable samples and well-defined instruments could help to validate our findings.

\section{Acknowledgments}

This study was supported by National Natural Science Foundation of China (31371024) and Qingdao Outstanding Health Professional Development Fund.

\section{Disclosure of Interests}

The authors declare no conflict of interest.

\section{References}

Akaike, H. (1987). Factor analysis and AIC. Psychometrika, 52, 317-332.

Anstey, K. J., Windsor, T. D., Jorm, A. F., Christensen, H., \& Rodgers, B. (2004). Association of pulmonary function with cognitive performance in early, middle and late adulthood. Gerontology, 50, 230-234.

Becker, A., Busjahn, A., Faulhaber, H. D., Bahring, S., Robertson, J., Schuster, H., \& Luft, F. C. (1997). Twin zygosity. Automated determination with microsatellites. The Journal of Reproductive Medicine, 42, 260-266.

Cassarino, M., \& Setti, A. (2015). Environment as 'brain training': A review of geographical and physical environmental influences on cognitive ageing. Ageing Research Reviews, 23(Pt B), 167-182.

Chen, W. L., Peng, T. C., Sun, Y. S., Yang, H. F., Liaw, F. Y., Wu, L. W., ... Kao, T. W. (2015). Examining the association between quadriceps strength and cognitive performance in the elderly. Medicine (Baltimore), 94, e1335.

Duan, H., Ning, F., Zhang, D., Wang, S., Zhang, D., Tan, Q., ... Pang, Z. (2013). The qingdao twin registry: A status update. Twin Research and Human Genetics, 16, 79-85.

Emery, C. F., Finkel, D., \& Pedersen, N. L. (2012). Pulmonary function as a cause of cognitive aging. Psychological Science, 23, 1024-1032.

Emery, C. F., Pedersen, N. L., Svartengren, M., \& McClearn, G. E. (1998). Longitudinal and genetic effects in the relationship between pulmonary function and cognitive performance. The Journal of Gerontology, Series B, Psychology Sciences and Social Sciences, 53, P311-317.

Feng, L., Lim, M. L., Collinson, S., \& Ng, T. P. (2012). Pulmonary function and cognitive decline in an older Chinese population in Singapore. COPD, 9, 555-562.

Gluhm, S., Goldstein, J., Loc, K., Colt, A., Liew, C. V., \& CoreyBloom, J. (2013). Cognitive performance on the mini- mental state examination and the montreal cognitive assessment across the healthy adult lifespan. Cognitive and Behavioral Neurology, 26, 1-5.

Hagenaars, S. P., Harris, S. E., Davies, G., Hill, W. D., Liewald, D. C., Ritchie, S. J., ... Deary, I. J. (2016). Shared genetic aetiology between cognitive functions and physical and mental health in UK Biobank $(\mathrm{N}=112151)$ and 24 GWAS consortia. Molecular Psychiatry, 21, 1624-1632.

Johnson, W., Deary, I. J., McGue, M., \& Christensen, K. (2009a). Genetic and environmental links between cognitive and physical functions in old age. The Journal of Gerontology, Series B, Psychology Sciences and Social Sciences, 64, 65-72.

Johnson, W., Deary, I. J., McGue, M., \& Christensen, K. (2009b). Genetic and environmental transactions linking cognitive ability, physical fitness, and education in late life. Psychology and Aging, 24, 48-62.

Li, L., Gao, W., Lv, J., Cao, W., Zhan, S., Yang, H., \& Hu, Y. (2006). Current status of the Chinese national twin registry. Twin Research and Human Genetics, 9, 747-752.

Li, L., Gao, W., Yu, C., Lv, J., Cao, W., Zhan, S., ... Hu, Y. (2013). The Chinese national twin registry: An update. Twin Research and Human Genetics, 16, 86-90.

Luis, C. A., Keegan, A. P., \& Mullan, M. (2009). Cross validation of the montreal cognitive assessment in community dwelling older adults residing in the Southeastern US. International Journal of Geriatric Psychiatry, 24, 197201.

Mueller-Schotte, S., van der Schouw, Y. T., Bleijenberg, N., \& Schuurmans, M. J. (2016). Is visual function associated with cognitive activity engagement in middle-aged and elderly individuals? A cross-sectional study. Experimental Gerontology, 82, 104-111.

Nasreddine, Z. S., Phillips, N. A., Bedirian, V., Charbonneau, S., Whitehead, V., Collin, I., ... Chertkow, H. (2005). The montreal cognitive assessment, MoCA: A brief screening tool for mild cognitive impairment. Journal of the American Geriatrics Society, 53, 695-699.

Ohsugi, H., Murata, S., Kubo, A., Hachiya, M., Hirao, A., Fujiwara, K., \& Kamijou, K. (2014). Verification of the correlation between cognitive function and lower limb muscle strength for the community-dwelling elderly. Journal of Physical Therapy Science, 26, 1861-1863.

Pang, Z., Ning, F., Unger, J., Johnson, C. A., Wang, S., Guo, Q., ... Lee, L. (2006). The qingdao twin registry: A focus on chronic disease research. Twin Research and Human Genetics, 9, 758-762.

Rantanen, T., Avlund, K., Suominen, H., Schroll, M., Frandin, K., \& Pertti, E. (2002). Muscle strength as a predictor of onset of ADL dependence in people aged 75 years. Aging Clinical and Experimental Research, 14(Suppl. 3), 10-15.

Saito, Y., Sugawara, N., Yasui-Furukori, N., Takahashi, I., Nakaji, S., \& Kimura, H. (2013). Cognitive function and number of teeth in a community-dwelling population in Japan. Annals of General Psychiatry, 12, 20.

Sternang, O., Reynolds, C. A., Finkel, D., Ernsth-Bravell, M., Pedersen, N. L., \& Dahl Aslan, A. K. (2016). Grip strength and cognitive abilities: associations in old age. The Journal 
of Gerontology, Series B, Psychology Sciences and Social Sciences, 71, 841-848.

Tian, X., Xu, C., Wu, Y., Sun, J., Duan, H., Zhang, D., ... Tan, Q. (2017). Genetic and environmental influences on pulmonary function and muscle strength: The Chinese twin study of aging. Twin Research and Human Genetics, 20.

Tomsey, C. S., Kurtz, M., Kist, F., Hockensmith, M., \& Call, P. (2001). Comparison of powerplex 16, pow-
erPlex1.1/2.1, and ABI AmpfISTR profiler plus/cofiler for forensic use. Croatian Medical Journal, 42, 239243.

Xu, C., Sun, J., Ji, F., Tian, X., Duan, H., Zhai, Y., ... Tan, Q. (2015). The genetic basis for cognitive ability, memory, and depression symptomatology in middle-aged and elderly Chinese twins. Twin Research and Human Genetics, 18, 79-85. 